\title{
Ab initio nonequilibrium quantum transport and forces with the real-space projector augmented wave method
}

Chen, Jingzhe; Thygesen, Kristian S.; Jacobsen, Karsten W.

Published in:

Physical Review B Condensed Matter

Link to article, DOI:

10.1103/PhysRevB.85.155140

Publication date:

2012

Document Version

Publisher's PDF, also known as Version of record

Link back to DTU Orbit

Citation (APA):

Chen, J., Thygesen, K. S., \& Jacobsen, K. W. (2012). Ab initio nonequilibrium quantum transport and forces with the real-space projector augmented wave method. Physical Review B Condensed Matter, 85(15), 155140.

https://doi.org/10.1103/PhysRevB.85.155140

\section{General rights}

Copyright and moral rights for the publications made accessible in the public portal are retained by the authors and/or other copyright owners and it is a condition of accessing publications that users recognise and abide by the legal requirements associated with these rights.

- Users may download and print one copy of any publication from the public portal for the purpose of private study or research.

- You may not further distribute the material or use it for any profit-making activity or commercial gain

- You may freely distribute the URL identifying the publication in the public portal 


\title{
$A b$ initio nonequilibrium quantum transport and forces with the real-space projector augmented wave method
}

\author{
Jingzhe Chen, Kristian S. Thygesen, and Karsten W. Jacobsen* \\ Center for Atomic-scale Materials Design, Department of Physics, Technical University of Denmark, DK-2800 Kongens Lyngby, Denmark
}

(Received 15 March 2012; published 27 April 2012)

\begin{abstract}
We present an efficient implementation of a nonequilibrium Green's function method for self-consistent calculations of electron transport and forces in nanostructured materials. The electronic structure is described at the level of density functional theory using the projector augmented wave method to describe the ionic cores and an atomic orbital basis set for the valence electrons. External bias and gate voltages are treated in a self-consistent manner and the Poisson equation with appropriate boundary conditions is solved in real space. Contour integration of the Green's function and parallelization over $k$ points and real space makes the code highly efficient and applicable to systems containing several hundreds of atoms. The method is applied to a number of different systems, demonstrating the effects of bias and gate voltages, multiterminal setups, nonequilibrium forces, and spin transport.
\end{abstract}

DOI: 10.1103/PhysRevB.85.155140 PACS number(s): 31.15.-p, 71.15.-m, 72.10.Bg, 72.25.-b

\section{INTRODUCTION}

Electron transport across nanostructured interfaces is important in a range of different areas, including nanoelectronics, organic photovoltaics, and electrochemistry. First-principles modeling of electron transport at the nanoscale has so far mostly been applied to molecular junctions consisting of molecules contacted by metallic electrodes. ${ }^{1-5}$ However, more recent applications also include graphene nanoribbons, ${ }^{6-8}$ semiconducting and metallic nanowires, ${ }^{9-11}$ and bulk tunneling junctions for magnetoresistance and electrochemical applications. ${ }^{12,13}$ The rapid developments in these areas toward atomic-scale control of interface structures, and the continuing miniaturization of electronics components makes the development of efficient and flexible computational tools for the description of charge transport at the nanoscale an important endeavor.

The vast majority of first-principles electron transport studies have been based on density functional theory (DFT) within the local density (LDA) or generalized gradient approximations (GGA). This approach is in principle unjustified because the eigenvalues of the effective Kohn-Sham Hamiltonian do not represent the true quasiparticle energy levels. In particular, for tunneling junctions the energy gap between the highest occupied states and lowest unoccupied states is too small ${ }^{14,15}$ and this can lead to an overestimation of the conductance. More accurate calculations, including self-interaction corrections ${ }^{16}$ and more recently the many-body $G W$ approximation ${ }^{17-19}$ yield conductance values in better agreement with experiments. On the other hand, the nonequilibrium Green's function (NEGF)-DFT approach often provides a satisfactory qualitative description ${ }^{5,20}$ and its computational simplicity makes it a powerful tool for addressing nonequilibrium properties of complex systems. It should be mentioned that the formal problems associated with the use of DFT for transport are overcome by time-dependent DFT (TDDFT) which allows for an, in principle, exact description of the (longitudinal) current due to an externally applied field. ${ }^{21}$ However, it has been recently found that the standard TDDFT exchange-correlation potentials do not yield any improvement over the NEGF-DFT in terms of accuracy in predicting conductance. ${ }^{22}$
In addition to the electronic current, it is of interest to model the forces acting on the atoms under nonequilibrium conditions, i.e., under a finite bias voltage. Such forces ultimately determine the stability of current-carrying molecular devices, ${ }^{23,24}$ but can also be exploited to deliberately control the motion of single molecules by, e.g., injecting electrons into the molecular orbitals using a scanning tunneling microscope (STM).

In this paper we describe the implementation of the NEGF-DFT method in the grid-based projector augmented wave $(\mathrm{GPAW})^{25,26}$ electronic structure code. In GPAW the electronic states can be described either on a real-space grid or using an atomic orbital basis set. For the NEGF calculations, the Green's function is expanded in the atomic orbital basis while the Poisson equation is solved in real space. Contour integration and sparse matrix techniques, together with parallelization over both $k$ points and real space is exploited for optimal efficiency. Although the basic elements of our implementation are not new and have been described in earlier papers, ${ }^{27-30}$ the possibility of applying a general gate and/or finite bias voltage, the use of multiple leads, and inclusion of nonequilibrium forces on the ions provides a flexible and efficient computational platform for general purpose modeling of charge transport at the nanoscale and should be of interest to a large and growing community.

This paper is organized as follows. In Sec. II the transport model and formalism are introduced. In Sec. III we describe the numeric details including the complex contour integration technique used to obtain the nonequilibrium electron density from the Green's function, the use of sparse matrix methods, and the real-space solution of the Poisson equation. A number of illustrative applications are presented in Sec. IV. In Sec. V the computational details of these examples are listed, and Sec. VI is reserved for a brief summary.

\section{METHOD}

The transport model is shown in Fig. 1. Following the standard approach, the system is divided into left and right electrodes and the central scattering region (see the detailed description in the caption). The Hamiltonian of the system 


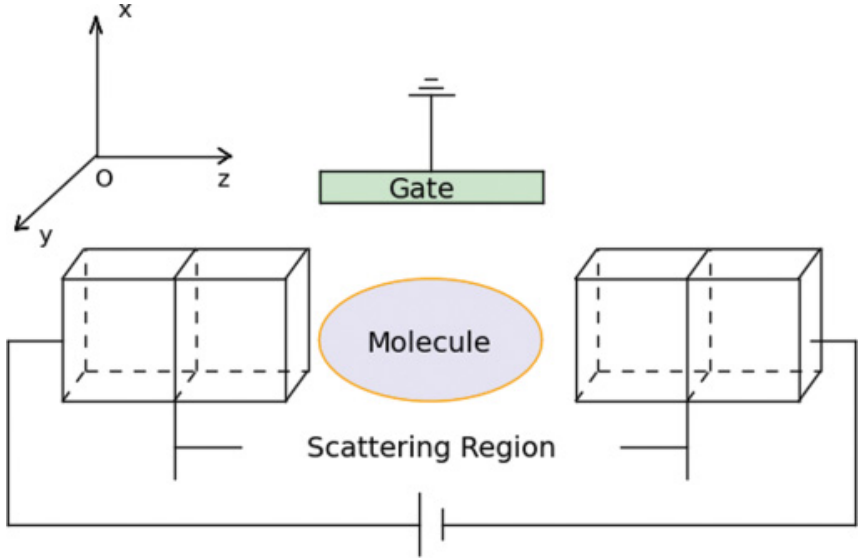

FIG. 1. (Color online) A scattering region including the nanostructure of interest (e.g., a molecule) and part of the electrode atoms is sandwiched between two semi-infinite electrodes. Periodic boundary conditions are used in the $x, y$ directions and open boundary conditions in the $z$ direction. The electron potential in the electrodes is periodic and can be obtained from a ground-state DFT calculation employing periodic boundary conditions in all directions. The Hartree potential at the scattering region boundary, which is used as boundary condition for the Poisson equation, is also obtained from the electrode calculation. The whole system can be subject to an external bias or gate voltage, and the electronic structure of the scattering region is calculated self-consistently in the presence of such external fields.

is given by (all notation related to PAW methodology is consistent with earlier GPAW papers ${ }^{25,26}$ )

$$
\tilde{\hat{H}}=-\frac{1}{2} \nabla^{2}+\tilde{v}_{\text {eff }}+\sum_{a i j}\left|\tilde{p}_{i}^{a}\right\rangle \Delta H_{i j}^{a}\left\langle\tilde{p}_{i}^{a}\right|,
$$

where $a$ denote the atoms in the system and $i, j$ label the PAW projector functions of a given atom. Using a (nonorthogonal) atomic orbital basis set, the Hamiltonian can be written in the following generic form:

$$
\left(\begin{array}{ccc}
H_{L L} & H_{L C} & 0 \\
H_{C L} & H_{C C} & H_{C R} \\
0 & H_{R C} & H_{R R}
\end{array}\right) .
$$

The "on-site" Hamiltonian matrices of the electrodes, $H_{L L}$ (left) and $H_{R R}$ (right), and the coupling matrices $H_{L C}$ and $H_{R C}$, can be obtained from a homogeneous bulk calculation. If a bias voltage $V$ is applied, the matrices corresponding to the left and right electrodes should be shifted by $e V$ relative to each other, e.g., $H_{L C} \rightarrow H_{L C}+e V S_{L C}$ and $H_{L L} \rightarrow H_{L L}+$ $e V S_{L L}$, where $S$ denotes the overlap matrix. We assume that there is no coupling between basis functions belonging to different electrodes. This assumption can be always satisfied by making the scattering region large enough.

The retarded Green's function is written as

$$
G^{r}(\varepsilon)=\left[(\varepsilon) S-H_{C C}-\Sigma_{L}^{r}(\varepsilon)-\Sigma_{R}^{r}(\varepsilon)\right]^{-1} .
$$

The self-energies $\Sigma_{L / R}^{r}$ represent the coupling to the electrodes and are obtained using the efficient decimation technique. ${ }^{31}$

The lesser Green's function is written as ${ }^{32}$

$$
G^{<}(\varepsilon)=G^{r}(\varepsilon) \Sigma^{<}(\varepsilon) G^{a}(\varepsilon)+\left(1+G^{r} \Sigma^{r}\right) G_{0}^{<}\left(1+\Sigma^{a} G^{a}\right) .
$$

The latter term is nonzero for truly bound states and vanishes for states acquiring any width.

The pseudodensity matrix (for the pseudowave in the PAW framework) is the integral of $G^{<}$,

$$
\begin{aligned}
D= & \frac{1}{2 \pi i} \int_{C}\left[G^{r}(z)-G^{a}(z)\right] d z+\frac{i}{2 \pi} \int_{E_{f}-e V / 2}^{E_{f}+e V / 2} G^{<}\left(\varepsilon^{\dagger}\right) d \varepsilon \\
& -2 \pi \theta i \sum_{i} G^{r}\left(\epsilon_{i}\right) .
\end{aligned}
$$

Here $\theta=k_{B} T$ and $C$ is a contour for the integral to be discussed further in Sec. III.

The nonequilibrium electron density is obtained as

$$
\tilde{n}(\mathbf{r})=\sum_{\nu \mu} D_{\nu \mu} \Phi_{\nu}(\mathbf{r})^{*} \Phi_{\mu}(\mathbf{r})+\sum_{a} \tilde{n}_{c}^{a}
$$

where $\Phi_{\nu}$ is an atomic orbital basis function and $\tilde{n}_{c}^{a}$ is the atomic pseudocore density. As is standard in the PAW formalism a tilde indicates a smooth quantity as opposed to an all-electron quantity. The smooth charge density is given by

$$
\tilde{\rho}(\mathbf{r})=\tilde{n}(\mathbf{r})+\sum_{a} \sum_{\ell m} Q_{\ell m}^{a} \hat{g}_{\ell m}^{a}(\mathbf{r})
$$

where $Q_{\ell m}^{a}$ are multipole moments and $\hat{g}_{\ell m}^{a}(\mathbf{r})$ is a so-called shape function. The last term is the contribution to the charge density coming from the positively charged nuclei.

The effective potential is found as

$$
\tilde{v}=\tilde{v}_{\text {coul }}+\tilde{v}_{\mathrm{xc}}+\sum_{a} \bar{v}^{a},
$$

where the Coulomb potential satisfies the Poisson equation $\nabla^{2} \tilde{v}_{\text {coul }}=-4 \pi \tilde{\rho}$, while $\tilde{v}_{\text {xc }}$ and $\bar{v}^{a}$ are the exchangecorrelation potential and zero potential, respectively. $\bar{v}^{a}$ is a parameter chosen to smoothen $\tilde{v}$ and which vanishes outside the augmentation sphere of atom $a .^{33}$

To obtain self-consistency we thus have the iteration process $D \rightarrow \rho \rightarrow V_{\text {eff }} \rightarrow H \rightarrow D \rightarrow \cdots$. After convergence the current can be calculated by

$$
\begin{aligned}
I(V)= & \frac{1}{\pi} \int_{-\infty}^{\infty}\left[f_{L}(\varepsilon)-f_{R}(\varepsilon)\right] \operatorname{Tr}\left[\Gamma_{L}(\varepsilon) G^{r}(\varepsilon)\right. \\
& \left.\times \Gamma_{R}(\varepsilon) G(\varepsilon)^{\dagger}\right] d \varepsilon,
\end{aligned}
$$

where $\Gamma_{L / R}(\varepsilon)=i\left(\Sigma_{L / R}^{r}(\varepsilon)-\Sigma_{L / R}^{r}(\varepsilon)^{\dagger}\right)$. For a derivation of the current formula we refer the reader to Ref. 34 (orthogonal basis) or Ref. 35 (nonorthogonal basis).

The nonequilibrium force is obtained from the derivative of the total energy with respect to atomic positions. In the PAW framework, the total energy is written

$$
E=\tilde{E}+\sum_{a}\left(E^{a}-\tilde{E}^{a}\right),
$$

with

$$
\begin{aligned}
\tilde{E}= & \sum_{\nu \mu} \rho_{\nu \mu}\left\langle\Phi_{\mu}\left|-\frac{1}{2} \nabla^{2}\right| \Phi_{\nu}\right\rangle+\frac{1}{2} \int \frac{\tilde{\rho}(\mathbf{r}) \tilde{\rho}(\mathbf{r})^{\prime}}{\left|\mathbf{r}-\mathbf{r}^{\prime}\right|} d \mathbf{r} d \mathbf{r}^{\prime} \\
& +\sum_{a} \int \tilde{n}(\mathbf{r}) \bar{v}^{a}(\mathbf{r}) d \mathbf{r}+E_{\mathrm{xc}}[\tilde{n}] .
\end{aligned}
$$


The force can be obtained as

$$
\mathbf{F}^{a}=-\frac{\partial E}{\partial \mathbf{R}^{a}},
$$

where

$$
\begin{aligned}
\frac{\partial E}{\partial \mathbf{R}^{a}}= & \sum_{\nu \mu} \frac{\partial E}{\partial \rho_{\nu \mu}} \frac{\partial \rho_{\nu \mu}}{\partial \mathbf{R}^{a}}+\sum_{\nu \mu} \frac{\partial E}{\partial T_{\nu \mu}} \frac{\partial T_{\nu \mu}}{\partial \mathbf{R}^{a}} \\
& +\sum_{L} \int \frac{\delta E}{\delta \tilde{g}_{L}^{a}(\mathbf{r})} \frac{d \tilde{g}_{L}^{a}(\mathbf{r})}{d \mathbf{R}^{a}} d \mathbf{r} \\
& +\int \frac{\delta E}{\delta \tilde{n}(\mathbf{r})} \frac{\partial \tilde{n}(\mathbf{r})}{\partial \mathbf{R}^{a}} d \mathbf{r}+\int \frac{\delta E}{\delta \bar{v}^{a}(\mathbf{r})} \frac{d \bar{v}^{a}(\mathbf{r})}{d \mathbf{R}^{a}} d \mathbf{r} \\
& +\sum_{b i j} \frac{\partial E}{\partial D_{i j}^{b}} \frac{\partial D_{i j}^{b}}{\partial \mathbf{R}^{a}} .
\end{aligned}
$$

We note that the expression given above does not include the recently discussed Berry phase contributions to the nonequilibrium force. ${ }^{24}$

\section{NUMERICAL DETAILS}

\section{A. Contour integration technique}

The contour for the Green's function integral in Eq. (5) is shown in Fig. 2. The retarded and lesser Green's functions are integrated along the path $\mathrm{AB}$ (see Fig. 2) in the upper half plane and the path EF closely above the real axis in the bias window. ${ }^{29,36}$

For the retarded Green's function we use Gaussian quadrature by which a precision corresponding to a $2 N-1$ order polynomial can be obtained by $N$ points. We use an adaptive method to find the energy points necessary to obtain a sufficient precision: ${ }^{37}$ For a given region $[c-h, c+h]$, the integral $Q$ of

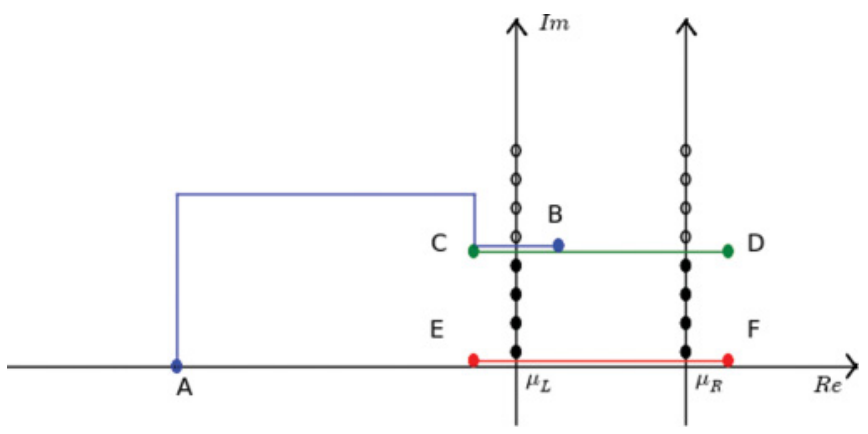

FIG. 2. (Color online) The contour used for the Green's function integral in the complex energy plane. The coordinates of the indicated points are as follows: $\mathrm{A}\left(E_{\min }, 0\right)$, where $E_{\min }$ is less than all the eigenenergies of the system, which is usually taken as $\mu_{L}-100 \mathrm{eV}$ since we only calculate the valence electrons states; B $\left(\mu_{L}+m k_{B} T, \Delta\right)$, where $m$ satisfies $e^{-m} \approx 0$ (a typical value of $m$ is 10), $k_{B}$ is the Boltzmann constant, $T$ is the electron temperature, $\Delta$ is normally between $\epsilon_{n}$ and $\epsilon_{n+1}$, where $\epsilon_{i}=(2 i-1) \pi$ (with $i$ a positive integer) is a pole of the Fermi-Dirac distribution function, thus the singulars below $\Delta$ should be counted when summing up the residues; $\mathrm{C}\left(\mu_{L}-m k_{B} T, \Delta\right)$; $\mathrm{D}\left(\mu_{R}+m k_{B} T, \Delta\right) ; \mathrm{E}\left(\mu_{L}-m k_{B} T, \eta\right)$, $\eta$ is infinitesimal to avoid the inversion divergence; $\mathrm{F}\left(\mu_{R}+m k_{B} T, \eta\right)$. a function $f$ can be estimated with the Gauss-Lobatto formula,

$$
\begin{aligned}
Q= & \frac{h}{6}\left[f(c-h)+5 f\left(c-\frac{1}{\sqrt{5}} h\right)+5 f\left(c+\frac{1}{\sqrt{5}} h\right)\right. \\
& +f(c+h)]
\end{aligned}
$$

and furthermore a Kronrod formula can be used to estimate the precision of the integral ${ }^{38}$

$$
\begin{aligned}
Q^{\prime}= & \frac{h}{1470}\left[\left(77 f(c-h)+432 f\left(c-\sqrt{\frac{2}{3}} h\right)\right.\right. \\
& +625 f\left(c-\frac{1}{\sqrt{5}} h\right)+672 f(c) \\
& +625 f\left(c+\frac{1}{\sqrt{5}} h\right)+432 f\left(c+\sqrt{\frac{2}{3}} h\right) \\
& +77 f(c+h)] .
\end{aligned}
$$

The difference between $Q$ and $Q^{\prime}$ can be taken as the precision of the integral.

The adaptive procedure to get the integral of the Green's function in a region $[c-h, c+h]$ is as follows: (1) Calculate $Q$ and $Q^{\prime}$, then compare the difference $\Delta$ with the tolerance $\delta$. (2) If $\Delta$ is smaller than $\delta$, the integral is converged and $Q$ is used as integral result. If not, divide the region [ $c-$ $h, c+h]$ into three subregions $\left[c-h, c-\frac{1}{\sqrt{5}} h\right],\left[c-\frac{1}{\sqrt{5}} h, c+\right.$ $\left.\frac{1}{\sqrt{5}} h\right],\left[c+\frac{1}{\sqrt{5}} h, c+h\right]$ and redo step (1) for each subregion until the integral is converged in the whole region.

For the lesser Green's function inside the bias window, we use the simple composite trapezoidal rule to obtain the integral. However, numerical errors can easily occur close to the real axis where the Green's function has singularities. For this reason we apply the double-contour method introduced in Ref. 29: First, the integral of the retarded Green's function is calculated along the path CD (Fig. 2) above the bias window, which is the spectrum for all the electronic states in the bias window $S$, then both electron spectrum $G^{<}$and hole spectrum $G^{>}$are integrated along the path $\mathrm{EF}$, and we have $S=D_{e}+$ $D_{h}$ according to the definition of the Green's function, where electron density $D_{e}$ and hole density $D_{h}$ are obtained from the integral of $G^{<}$and $G^{>}$, respectively. The numeric error, $\Delta S=S-D_{e}-D_{h}$ is normally a nonzero quantity due to the integral insufficiency. As a correction, the error is distributed to $D_{e}$ and $D_{h}$ by the matrix element weight.

\section{B. Sparse matrix handling}

Because the matrix inversion cost scales as $N^{3}$, where $N$ is the dimension of the matrix, the matrix inversion turns out to be the main computational cost for large systems. Hence a sparse matrix method is implemented to obtain the Green's function.

We define a quenching layer as a slab whose left side has no overlap with the right side due to the finite cutoff in the range of the atomic orbitals. Hence an overlap or Hamiltonian matrix can be split into several blocks, with each block representing the on-site values of a quenching layer or the coupling between 
two adjacent quenching layers. Note that quenching layers here are different from the principal layers used in the transport framework, where the latter is supposed to be repeatable as well.

Physical quantities such as density or transmission are often determined by fairly few blocks of a matrix. To see this consider the simple example of a two-probe system. In this case, the scattering region is divided into five quenching layers. Figure 3 shows the sparse matrix structure of the overlap or the Hamiltonian matrix. The blocks outside the scattering region are from electrode calculations and are always fixed.

First we discuss how to obtain the real-space pseudodensity which can be obtained by a projection of the pseudodensity matrix as in Eq. (6). We see that if the states $\Phi_{\nu}(\mathbf{r})$ and $\Phi_{\mu}(\mathbf{r})$ have no overlap, the contribution from the pseudodensity matrix is zero, i.e., the white blocks in Fig. 3(a) do not affect the density. So when we calculate the density matrix from the integral of the Green's function, only the blue and green blocks in the Green's function matrix [Fig. 3(a)] are necessary. There are really two different parts, because two types of Green's functions are involved when calculating the density matrix: the equilibrium part and the nonequilibrium part. We need the blue and green blocks of the retarded Green's function for the former and of the Keldysh Green's function for the latter. Through Eq. (4) and the finite extent of the self-energy matrix, which is only nonzero in the principle layers close to the electrodes, we see that the blue and green blocks in Fig. 3 in the Keldysh Green's function matrix can be obtained from only the red blocks of the retarded Green's function [Fig. 3(c)]. So when we do the matrix inversion to calculate the retarded Green's function by Eq. (3), the red blocks in Fig. 3(a) are necessary for energy points on the path EF in Fig. 2, and the blue and green blocks in Fig. 3(c) are necessary for energy points on the other path segments in Fig. 2.

We can also see that the red and orange blocks in Fig. 3(b) are needed to calculate the density of states (DOS) by $\operatorname{DOS}(\varepsilon)=-\frac{1}{\pi} \operatorname{Im}\left[\operatorname{Tr}\left(G\left(\varepsilon^{+}\right) S\right]\right.$ and the pink blocks in Fig. 3(b) are needed to calculate the transmission function $T(\varepsilon)=$ $\operatorname{Tr}\left[\Gamma_{L}(\varepsilon) G\left(\varepsilon^{+}\right) \Gamma_{R}(\varepsilon) G\left(\varepsilon^{+}\right)^{\dagger}\right]$.

The formulas below provide a quick solution for the necessary blocks. Here we just consider this particular matrix (shown in Fig. 3) as an example to show how the method works. A general formalism, which works for arbitrary number of electrodes and arbitrary number of principal layers in each electrode, is introduced in Ref. 39.

First, the central block $N$ in Fig. 3(b) of the retarded Green's function can be solved through the equations

$$
\begin{gathered}
Q_{2}^{L}=L_{22}^{-1}, \quad Q_{1}^{L}=\left(L_{11}-L_{12} Q_{2}^{L} L_{21}\right)^{-1} \\
Q_{2}^{R}=R_{22}^{-1}, \quad Q_{1}^{R}=\left(R_{11}-R_{12} Q_{2}^{R} R_{21}\right)^{-1}, \\
N=\left(M-\sum_{J=L, R} J_{12} Q_{2}^{J} J_{21}\right)^{-1}
\end{gathered}
$$

where $L_{i j}$ and $R_{i j}$ are the blocks shown in Fig. 3(a) representing the matrix $\varepsilon S-H_{C C}-\Sigma_{L}^{r}(\varepsilon)-\Sigma_{R}^{r}(\varepsilon)$. Then, for the remaining blocks of the retarded Green's function matrix, we have to iterate the formulas

$$
\begin{aligned}
G_{i, i}^{L} & =Q_{i}^{L}\left(I-L_{i, i-1} G_{i-1, i}^{L}\right), \\
G_{i, i}^{L} & =\left(I-G_{i, i-1} L_{i-1, i}^{L}\right) Q_{i}^{L}, \\
G_{i, j}^{L} & =-Q_{i} L_{i, i-1} G_{i-1, j}^{L}, \\
G_{j, i}^{L} & =-G_{j, i-1}^{L} L_{i-1, i} Q_{i}^{L} \quad(j<i),
\end{aligned}
$$

where $G_{i, j}^{L}$ is the block from the central part to electrode $L$ and $G_{0,0}^{L}$ is $N$ in Eq. (18), the blocks from the central part to electrode $R$ have a similar solution. For all the required blocks, a quick solution can be obtained using a combination of the recursive formulas (18). If we denote the number of quenching layers by $n$, the computational cost is roughly given by $n$ times the cost of an inversion operation plus $4 n$ times the cost of matrix multiplication. ${ }^{39}$

\section{Fixed boundary conditions}

The electronic potential of the metal electrodes will usually be very efficiently screened so that after only a few atomic layers into the electrodes we can assume that the potential is equal to the equilibrium potential plus a possible constant bias potential, which is normally $\mathrm{eV} / 2$ for the cathode and $-\mathrm{eV} / 2$ for the anode ( $\mathrm{V}$ stands for the bias voltage). We shall apply open boundary conditions (in contrast to, say, periodic ones) where the bias is applied by fixing the potential values at the boundaries before solving the Poisson equation. ${ }^{40}$ This procedure also allows for a net charge in the scattering region, in which case the perturbation of the electron potential into the electrodes will of course be somewhat more long ranged.

The Poisson equation $\nabla^{2} \tilde{v}_{\text {coul }}=-4 \pi \tilde{\rho}$ is solved in reciprocal space in the $x$ and $y$ directions while it is solved in real space in the $z$ coordinate, i.e., in the the transport direction. Mathematically we have

$$
\left(\frac{d^{2}}{d z^{2}}-\vec{G}^{2}\right) \tilde{v}_{\text {coul }}(z, \vec{G})=-4 \pi \tilde{\rho}(z, \vec{G}),
$$

where $\vec{G}$ is the vectors of the two-dimensional (2D) real grids used for the Fourier transformation.

Equation (19) is solved by the sparse matrix linear equation subroutine provided by the LAPACK package. An advantage of this Poisson equation solution is the good parallelization behavior. The 2D Fourier transformations are independent for the different real-space slices, and the linear equations (19) can be solved independently for different $\vec{G}$ vectors.

\section{RESULTS}

\section{A. Quantum capacitor}

We consider a simple capacitor system consisting of two semi-infinite $\mathrm{Na}$ electrodes separated by a vacuum gap (see the upper part of Fig. 4). When a bias voltage is applied to the system, electrons accumulate or deplete on the $\mathrm{Na}(100)$ surfaces. According to the classic parallel plate capacitor model, the surface charge should be

$$
Q_{\mathrm{cl}}=\varepsilon_{0} V A / d,
$$




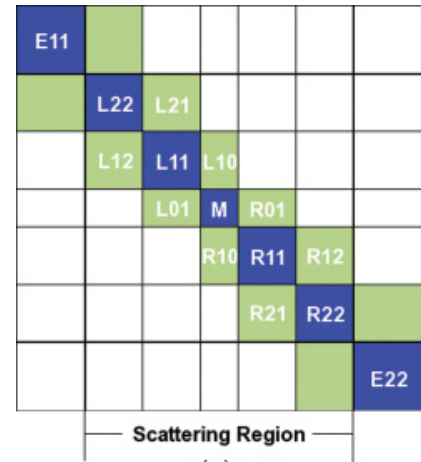

(a)

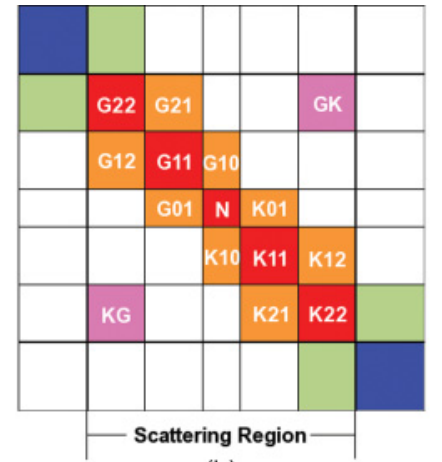

(b)

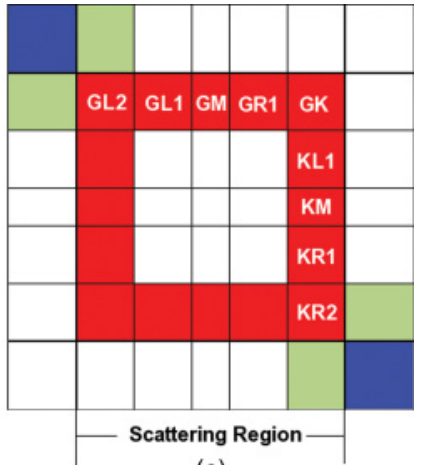

(c)

FIG. 3. (Color online) Schematic of the matrix blocks. (a) The Hamiltonian or overlap matrix: The blue and green blocks represent the on-site and coupling submatrices for the different quenching layers, respectively. (b) The Green's function matrix when evaluating the density, DOS, or transmission: The red and orange blocks represent the submatrices needed to calculate the density matrix or DOS, and pink blocks are for the transmission coefficient. (c) The red blocks in the retarded Green's function matrix are necessary when calculating the Keldysh Green's function.

where $\varepsilon_{0}, A, d$ are the vacuum permittivity, area cross section of the unit cell, and the gap distance, respectively. We integrate the induced charge density in real space and obtain the net charge accumulation $Q=0.45 e$, which is close to the result of classic theory $Q_{\mathrm{cl}}=0.55 e$. One difference is that, in the quantum theory, the charge decays from the surface into the bulk in an oscillatory fashion (Friedel oscillations), instead of being localized exactly on the surface as assumed in the classic model. We also note that at a distance of about four layers from the surface the values for both the potential and the charge are very close to their bulk values. Hence this calculation confirms the screening approximation, namely, that a few layers away from the surface or scattering region the potential has reached its bulk value. Finally, we note that the relatively high bias
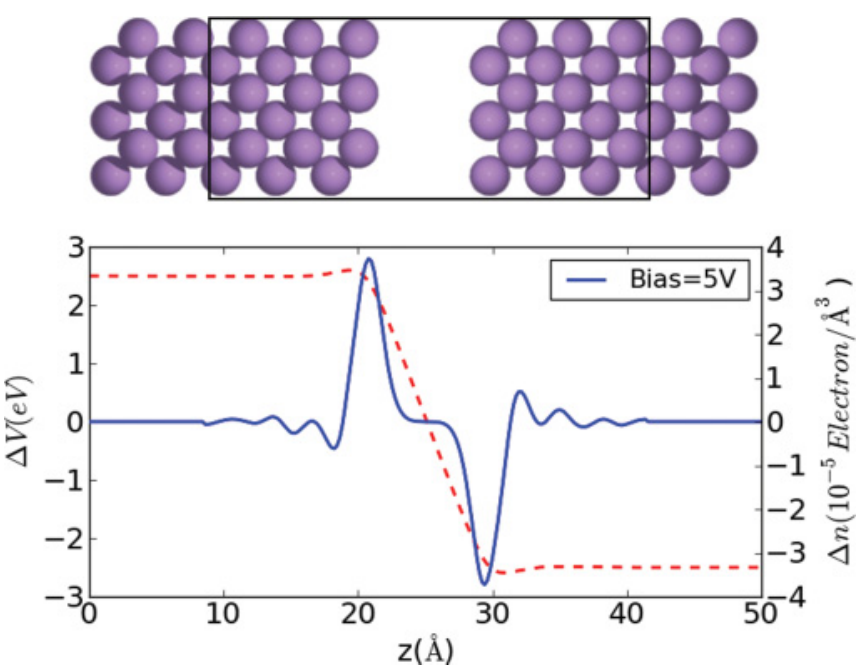

FIG. 4. (Color online) Upper panel: Capacitor model with the electrodes made of bcc sodium with the voltage drop along the (100) direction. The vacuum distance between the two electrodes is $8 \AA$, and the size of the unit cell in the transverse directions is $12.7 \AA \times$ $12.7 \AA$. The rectangle represents the scattering region. Lower panel: The nonequilibrium part of the electron density (solid blue) and the induced effective potential (dashed red) under a bias voltage of $5 \mathrm{~V}$, with the zero-bias values as the reference. The calculated values are averaged over the transverse plane. voltage of $\approx 5 \mathrm{~V}$ is possible in the present case where no current is flowing. On the other hand, the nonequilibrium states determining the current flow become increasingly difficult to calculate accurately for larger bias values due to the insufficient integral of the Keldysh Green's function in the bias window. As a consequence, electron transport calculations are typically possible or reliable up to bias voltages of around 2-3 V, depending on the transparency of the junction.

\section{B. Nonequilibrium forces}

The calculation of nonequilibrium forces is in principle a delicate problem involving nonconservative components. $^{23,24,41}$ For highly conducting molecular bridges an instability may occur which involves the Berry phase of the wave function. The description of such phenomena is beyond the scope of the usual NEGF + DFT framework, but in simpler cases, in particular, in cases with no or little current flow, the force expressions (11) and (12) still apply.

As an example, we show here a nonequilibrium force calculation for a $\mathrm{Au} / \mathrm{N}_{2} / \mathrm{Au}$ junction, where we can see the tendency toward molecular dissociation under a bias voltage. The structure (see the upper part of Fig. 5) is relaxed under zero bias until the maximum force is below $0.01 \mathrm{eV} / \AA$. When a positive bias is applied, electrons are redistributed over the molecule due to the electric field. Consequently, the two nitrogen atoms start to repel each other due to increased Coulomb repulsion which weakens the bond. The actual quantity of charge transfer to the molecule, which is about $0.01 e$ for $1-\mathrm{V}$ bias voltage on this system, shifts up the molecular energy spectrum, i.e., the energy levels follow the chemical potential of the left electrode (see the middle panel of Fig. 5). The force is mainly occurring only between the two nitrogen atoms, while there is no force induced between the electrode atoms and the $\mathrm{N}_{2}$ molecule. Equivalently, a negative bias voltage shifts down the levels and pull electrons out of the $\mathrm{N}_{2}$ molecule, and leads to an attractive force between the two nitrogen atoms.

\section{Electrostatic gate control}

One way of controlling the current flow through a nanoscale conductor is by electrostatic gating. This has been 

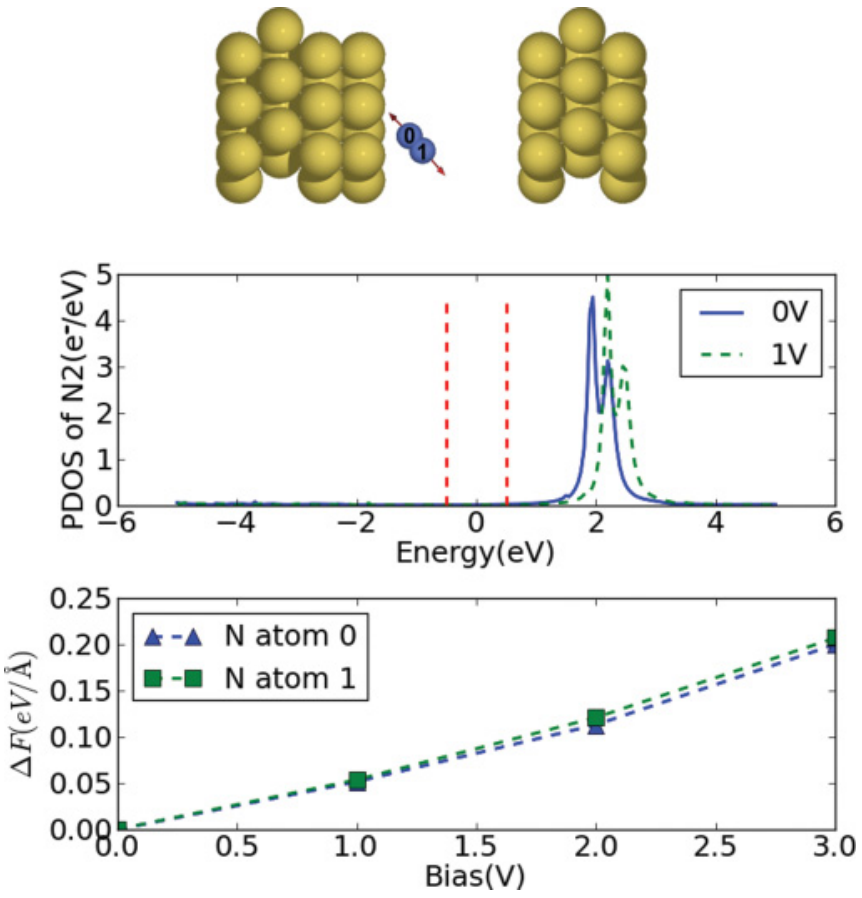

FIG. 5. (Color online) The bias voltage effect on a nitrogen molecule between two gold electrodes. Upper panel: The atomic structure of the system. The arrows represent the directions of the atomic forces generated by the bias voltage. Middle panel: PDOS (partial density of states) of the nitrogen molecule at 0 - and $1-\mathrm{V}$ bias voltage. The Fermi level is located at zero and the red dashed lines show the location of the bias window in the $1-\mathrm{V}$ case. Lower panel: The magnitude of the nonequilibrium atomic forces as a function of bias voltage.

demonstrated experimentally for graphene, where a metalinsulator transition was induced by gating, ${ }^{42,43}$ and for singlemolecule junctions where the individual electronic levels were moved in energy relative to the Fermi level of the source/drain electrodes. ${ }^{44,45}$ At the single molecule scale, the gate-molecule coupling is to a large extent determined by the device geometry with the screening effect playing an important role. ${ }^{46}$ For numerical simulations, the typical method of applying a gate is to add an external potential $v_{g}(\mathbf{r})$ to the effective potential of the system:

$$
\tilde{v}(\mathbf{r})=\tilde{v}^{0}(\mathbf{r})+v_{g}(\mathbf{r}) .
$$

We now consider the prototypical Au/benzene-1,4dithiolate/Au (Au-BDT-Au) junction (see the upper panel of Fig. 6) subject to three different gate potentials, $v_{g}(z)$ (see the middle panel of Fig. 6). We note that the structure of the $\mathrm{Au} / \mathrm{BDT}$ junction is presently being debated. ${ }^{47-49}$ However, for our purpose the simple model makes the sense.

In the lower part of Fig. 6 we plot the resulting effective gate potential $\Delta v(z)=\tilde{v}_{\mathrm{sc}}(z)-\tilde{v}_{\mathrm{sc}}^{0}(z)$, where the subscript sc denotes self-consistency, and the superscript 0 denotes zero gate voltage. Due to the screening in the metal, the effective gate potentials only affect the molecule region, and the narrower gate potential is seen to be less influenced by the self-consistency because it does not induce considerable charge transfer at the metal surfaces-a charge transfer that
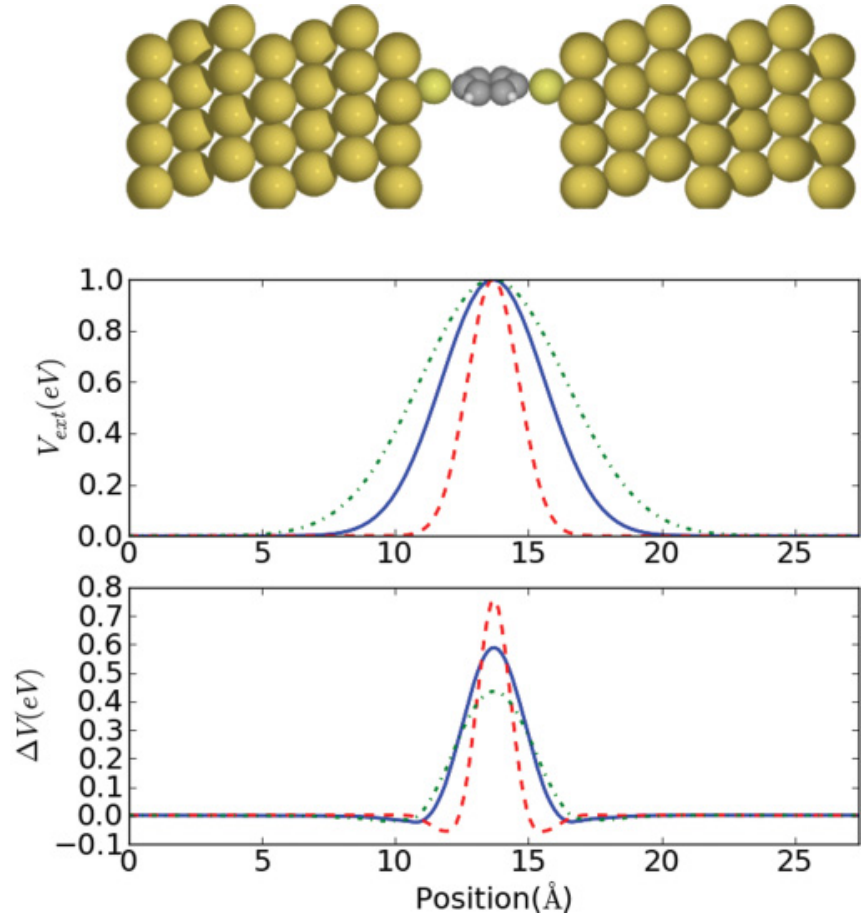

FIG. 6. (Color online) Effect of electrostatic gating of a benzene1,4-dithiolate molecule between two gold fcc(111) electrodes. Upper panel: The atomic structure of the system. Middle panel: The applied external potential. Lower panel: The effective additional potential after self-consistency.

otherwise tends to reduce the gate effect on the molecule. We note that the gate efficiency factor, $\left.\alpha=\Delta v_{(} z\right) / v_{g}(z)$, for these three potentials are about $0.8,0.6$, and 0.4 in the molecular region, with the larger efficiency obtained for the more localized gate potential. The value of 0.4 obtained for the most delocalized potential is fairly close to an experimental study $^{45}$ of the Au-BDT-Au system where an efficiency factor of 0.25 was reported.

In the following we illustrate how the gate voltage can be used to tune the conductance of a molecular junction. It has recently been shown that the transport through the molecule anthraquinone is strongly suppressed due to destructive quantum interference occurring close to the Fermi level when the molecule is connected to metallic electrodes. ${ }^{50,51}$ The quantum interference leads to a dip in the transmission function inside the energy gap between the highest occupied (HOMO) and lowest unoccupied (LUMO) molecular orbitals. Hence a large on-off ratio is expected when shifting the molecular energy levels by an external gate voltage. The upper panel of Fig. 7 shows the molecule connected to two gold fcc(111) surfaces through Au-S bonds. The effective potential with the gate voltage $-2 \mathrm{~V}$ is shown in the middle panel. We see that the potential of the central part of the anthraquinone molecule is shifted less than the potential for the outer parts of the molecule. This is due to the fact that different parts of the molecule polarize differently as a consequence of the detailed electronic structure. The HOMO is, for example, mainly localized at the connecting wires. The lower panel of Fig. 7 shows the change of transmission coefficient when a gate voltage of $-2 \mathrm{~V}$ is applied. Due to the characteristic 

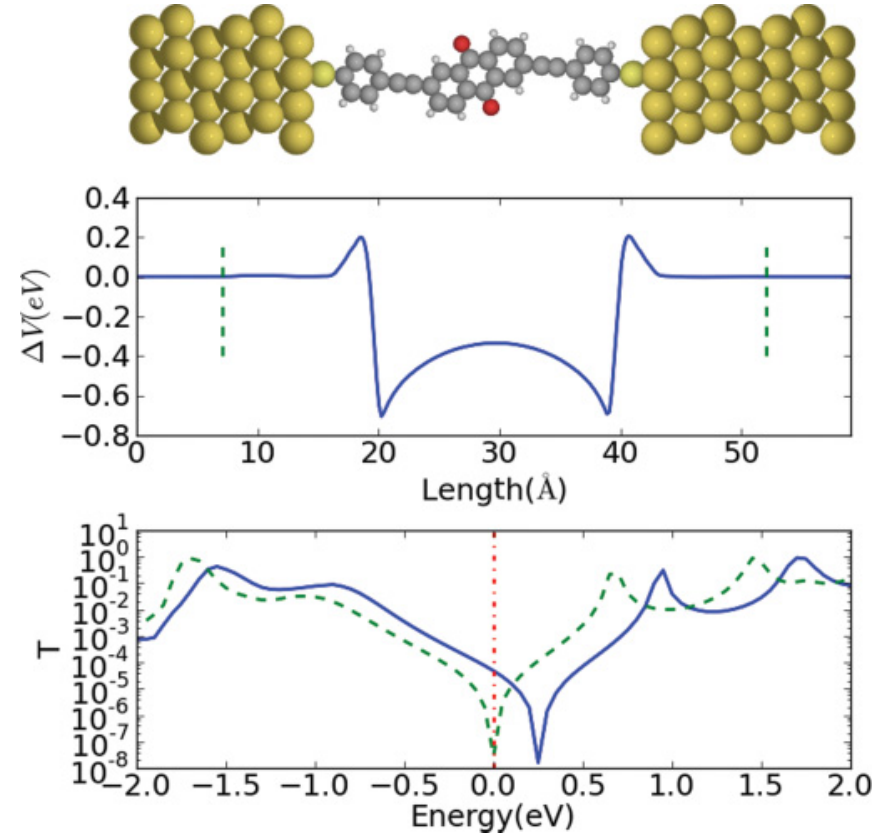

FIG. 7. (Color online) Gate tuning the conductance of a molecular transistor. Upper panel: The Au-anthraquinone-Au structure. Middle panel: The gate effect on the potential. Lower panel: The transmission coefficient at 0 and $-2 \mathrm{~V}$ gate voltages.

interference dip in the transmission a large on-off ratio of about a factor of 1000 is achieved. Note that the relatively poor gate efficiency of around 0.1 is due to Fermi-level pinning of the HOMO.

\section{Spin transport}

In this section, we investigate the nonequilibrium-driven magnetic transition in the spin transport in a zigzag graphene nanoribbon (ZGNR) which is proposed in Ref. 52 based on tight-binding calculations. The ZGNR's edge is spin polarized and it has an antiferromagnetic spin configuration if its number of atomic layers is even. ${ }^{53} \mathrm{~A}$ gap of about $1 \mathrm{eV}$ is opened between the different spin states and makes the ZGNR a semiconductor. It was noticed by Denis et al. that the ZGNR's magnetic ordering is killed when the external bias voltage exceeds the size of the gap. ${ }^{52}$ Here we reproduce this result for the graphene/ZGNR/graphene system shown in the upper panel of Fig. 8, where a ZGNR $(n n=8)$ is sandwiched between two semi-infinite graphene flakes. Under zero bias the PDOS of the central $\mathrm{C}$ atom along the ZGNR edge shows two peaks above and below the Fermi level, corresponding to the different spin directions (the middle panel in Fig. 8). The distance between the two peaks is about $1.6 \mathrm{eV}$, and equals the band gap. When this bias voltage reaches $1.0 \mathrm{~V}$, the current starts to increase (see Fig. 9). At bias voltage $2.0 \mathrm{~V}$ the edge magnetic moment disappears very abruptly, and the current starts to increase even faster.

Interestingly, in the tight-binding calculations presented in Ref. 52, both the magnetic moment and the current show a very abrupt feature at the bias threshold, while in our calculation, the current increases rather smoothly. This can be explained by the nonequilibrium potential in the $a b$ initio calculation leads
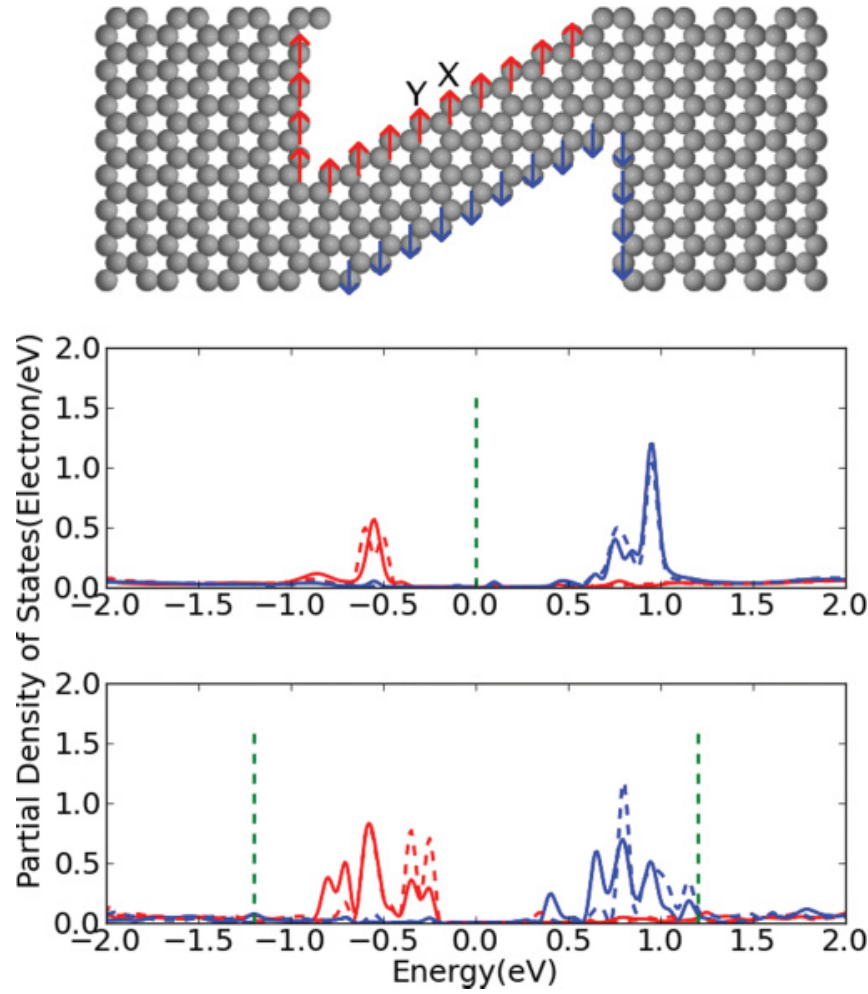

FIG. 8. (Color online) Spin transport in a zigzag graphene nanoribbon (ZGNR) bridge connecting to two semi-infinite graphene flakes. Upper panel: The atomic structure of the graphene/ZGNR/graphene system. Middle panel: The PDOS of a $\mathrm{C}$ atom at the ribbon's zigzag edge under zero bias; the red and blue solid lines represent the spin-up and spin-down PDOS of the $\mathrm{C}$ atom at the center of the zigzag edge (marked with $\mathrm{X}$ ), the red and blue dashed lines represent the spin-up and spin-down PDOS of the $\mathrm{C}$ atom next to the previous one at the zigzag edge (marked with Y), and the green dashed line is the Fermi level. Lower panel: The PDOS of a $\mathrm{C}$ atom at the ribbon's zigzag edge under bias voltage $V=2.4 \mathrm{~V}$.

to a rehybridization and broadening of the spectral peaks (see the lower panel of Fig. 8). We also note that in our calculation the disappearance of the magnetic moment is due to the two

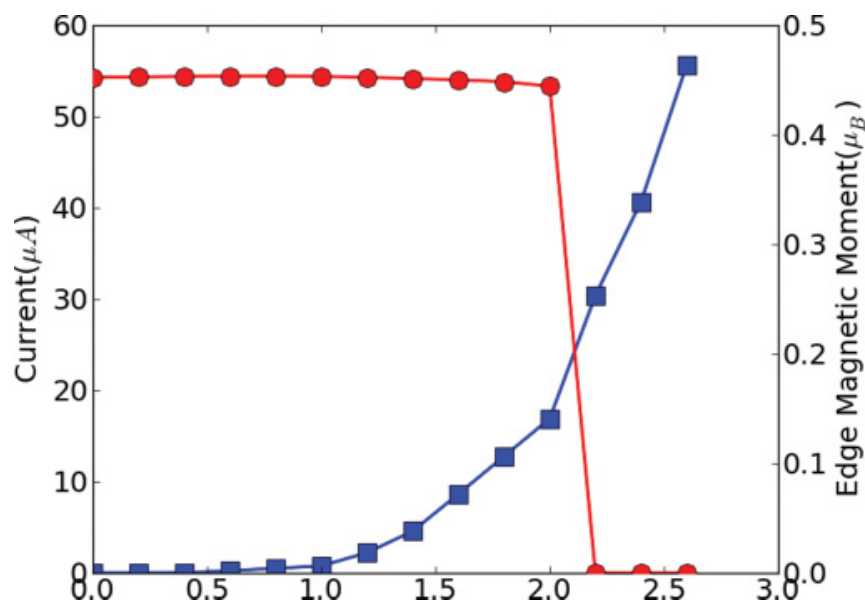

FIG. 9. (Color online) Calculated current (blue squares) and edge magnetic moment per $\mathrm{C}$ atom (red circles) as a function of bias voltage for the ZGNR structure shown in Fig. 8. 

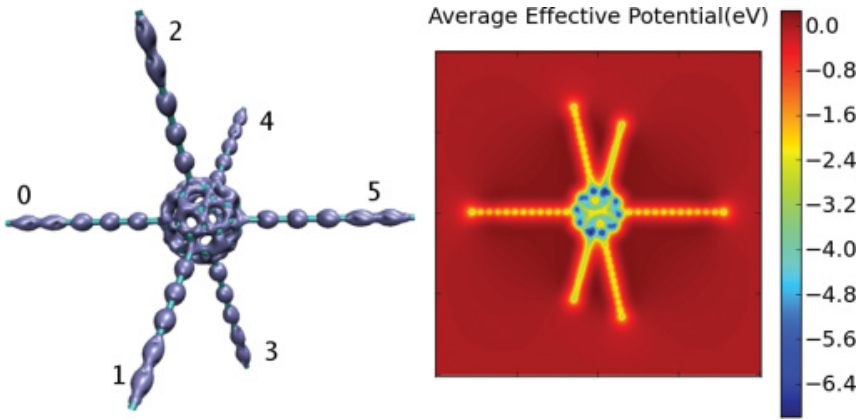

FIG. 10. (Color online) Left: The $\mathrm{C}_{60}-6$-terminal structure and real-space DOS at the Fermi level. Right: The averaged effective potential projected onto a plane cutting through the $\mathrm{C}_{60}$ molecule.

Stoner peaks moving into the bias window being half occupied, which is different from the complete band collapse in Ref. 52; this is because our ZGNR is not long enough. We can see from the lower panel of Fig. 8 that the gap shrinks more for the $\mathrm{C}$ atom further from the contact.

\section{E. Multiterminal transport}

The expression for the Green's function of the scattering region Eq. (3) can be straightforwardly extended to a multiterminal situation

$$
G^{r}(\varepsilon)=\left[\varepsilon S-H_{C C}-\sum_{J} \Sigma_{J}^{r}(\varepsilon)\right]^{-1},
$$

where $J$ is the index of the terminals. In contrast to the situation for a two-probe calculation, a zero boundary condition is applied for the effective potential for a multiterminal system, and buffer atoms are used to represent semi-infinite leads. This approach to multiterminal transport has been previously investigated in Ref. 54. It should be noted that the self-energy of a lead has to be "rotated" by an orthogonal transformation when the lead is not along either the $x, y$, or $z$ axes.

As an example, we consider a $\mathrm{C}_{60}$ molecule connected to six linear carbon atomic chains. Figure 10 (left) shows the projected DOS in real space evaluated at the Fermi level. The coverage suggests that the scattering states are itinerant in the whole system and the contact between the carbon chain and the $\mathrm{C}_{60}$ moelcule is transparent. Figure 10 (right) shows a $2 \mathrm{D}$ averaged potential in a plane cutting through the $\mathrm{C}_{60}$ molecule.

A matrix indicating the transmission at the Fermi level between the different leads is shown in Fig. 11. The matrix

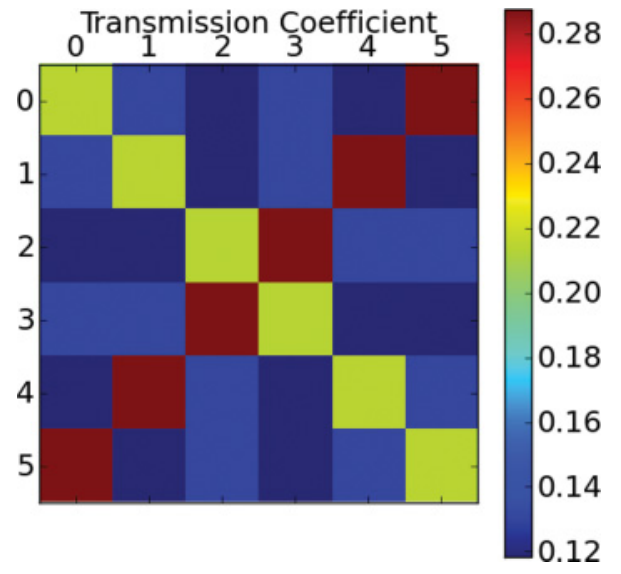

FIG. 11. (Color online) The transmission function evaluated at the Fermi energy between the six leads. The element $(i, j)$ refers to the transmission from lead $i$ to lead $j$; the diagonal element is the reflection coefficient.

index notation represents the lead number as shown in Fig. 10 (left). In particular, the diagonal corresponds to backscattering, i.e., it gives the reflection probability. We can see that electrons are more easily transmitted between leads opposing each other, whereas the transmission decreases if the electron has to turn an angle during the scattering process. This intuitive phenomenon can be explained by the quantum interference of the different partial waves. For the straightforward scattering, the quantum phases are the same for all the paths passing through the $\mathrm{C}_{60}$ molecule, and the electron therefore attains the greatest transmission probability. ${ }^{51}$

\section{COMPUTATIONAL DETAILS}

For completeness we list the key input parameters and CPU timings for the examples presented in this paper in Table I.

\section{SUMMARY}

We have described the implementation of the NFGF + DFT transport method in the GPAW code and illustrated its performance through application to a number of different molecular junctions. The electronic structure is described within the PAW methodology which provides all-electron accuracy at a computational cost corresponding to pseudopotential calculations. The Green's functions are represented in a basis set consisting of atomiclike orbitals while the Poisson equation with appropriate boundary conditions is solved in real space. The

TABLE I. Key parameters and CPU timings for the examples considered in this article.

\begin{tabular}{lcccccc}
\hline \hline System & $k$ sampling & Functional & Basis & Lead $k$ sampling & Processor number & Wall time (h) \\
\hline Capacitor & $(4,4)$ & LDA & SZ & $(4,4,15)$ & 4 & 0.5 \\
Au/N $/$ Au & $(2,2)$ & PBE & DZP (SZP) & $(2,2,15)$ & 32 & 3 \\
Gate (BDT) & $(2,2)$ & PBE & DZP (SZP) & $(2,2,15)$ & 32 & 32 \\
Gate (anthraquinon) & $(2,2)$ & PBE & DZP (SZP) & $(2,2,15)$ & 32 & 32 \\
Graphene/ZGNR/graphene & $(2,1)$ & PBE & SZP & $(2,2,7)$ & & 2 \\
C $_{60}$ & $(1,1)$ & LDA & SZ & $(1,1,15)$ & \\
\hline \hline
\end{tabular}


code is parallelized over $k$ points and real-space domains and sparse matrix techniques are applied for maximal efficiency. The flexibility of the method was illustrated through examples demonstrating electron transport under finite bias voltage, effect of electrostatic gating, spin transport, nonequilibrium forces, and multiterminal leads.

\section{ACKNOWLEDGMENTS}

The authors thank Jens Jørgen Mortensen and Ask Hjorth Larsen for helpful discussions. The authors acknowledge support from the Danish Center for Scientific Computing through Grant No. HDW-1103-06. The Center for Atomic-scale Materials Design is sponsored by the Lundbeck Foundation. *kwj@fysik.dtu.dk

${ }^{1}$ R. H. M. Smit, Y. Noat, C. Untiedt, N. D. Lang, M. C. van Hemert, and J. M. van Ruitenbeek, Nature (London) 419, 906 (2002).

${ }^{2}$ D. Djukic, K. S. Thygesen, C. Untiedt, R. H. M. Smit, K. W. Jacobsen, and J. M. van Ruitenbeek, Phys. Rev. B 71, 161402 (2005).

${ }^{3}$ L. Venkataraman, J. E. Klare, I. W. Tam, C. Nuckolls, M. S. Hybertsen, and M. L. Steigerwald, Nano Lett. 6, 458 (2006).

${ }^{4}$ S. Y. Quek, L. Venkataraman, H. J. Choi, S. G. Louie, M. S. Hybertsen, and J. B. Neaton, Nano Lett. 7, 3477 (2007).

${ }^{5}$ C. Li, I. Pobelov, T. Wandlowski, A. Bagrets, A. Arnold, and F. Evers, J. Am. Chem. Soc. 130, 318 (2007).

${ }^{6}$ F. Muñoz-Rojas, J. Fernández-Rossier, and J. J. Palacios, Phys. Rev. Lett. 102, 136810 (2009).

${ }^{7}$ H. Şahin and R. T. Senger, Phys. Rev. B 78, 205423 (2008).

${ }^{8}$ F. D. Novaes, R. Rurali, and P. Ordejón, ACS Nano 4, 7596 (2010).

${ }^{9}$ T. Markussen, R. Rurali, X. Cartoixà, A.-P. Jauho, and M. Brandbyge, Phys. Rev. B 81, 125307 (2010).

${ }^{10}$ Y. Lee, K. Kakushima, K. Shiraishi, K. Natori, and H. Iwai, J. Appl. Phys. 107, 113705 (2010).

${ }^{11}$ T. Ono and K. Hirose, Phys. Rev. Lett. 94, 206806 (2005).

${ }^{12}$ I. Rungger, O. Mryasov, and S. Sanvito, Phys. Rev. B 79, 094414 (2009).

${ }^{13}$ J. Chen, J. S. Hummelshøj, K. S. Thygesen, J. S. Myrdal, J. K. Nørskov, and T. Vegge, Catal. Today 165, 2 (2011).

${ }^{14}$ M. Dell' Angela, G. Kladnik, A. Cossaro, A. Verdini, M. Kamenetska, I. Tamblyn, S. Y. Quek, J. B. Neaton, D. Cvetko, and A. Morgante et al., Nano Lett. 10, 2470 (2010).

${ }^{15}$ J. M. Garcia-Lastra, C. Rostgaard, A. Rubio, and K. S. Thygesen, Phys. Rev. B 80, 245427 (2009).

${ }^{16}$ C. Toher and S. Sanvito, Phys. Rev. Lett. 99, 056801 (2007).

${ }^{17}$ M. Strange, C. Rostgaard, H. Häkkinen, and K. S. Thygesen, Phys. Rev. B 83, 115108 (2011).

${ }^{18}$ M. Strange and K. S. Thygesen, Beilstein J. Nanotechnol. 2, 746 (2011).

${ }^{19}$ T. Rangel, A. Ferretti, P. E. Trevisanutto, V. Olevano, and G.-M. Rignanese, Phys. Rev. B 84, 045426 (2011).

${ }^{20}$ M. S. Hybertsen, L. Venkataraman, J. E. Klare, A. C. Whalley, M. L. Steigerwald, and C. Nuckolls, J. Phys.: Condens. Matter 20, 374115 (2008).

${ }^{21}$ G. Stefanucci and C. O. Almbladh, Europhys. Lett. 67, 14 (2004).

${ }^{22}$ C. Y. Yam, X. Zheng, G. H. Chen, Y. Wang, T. Frauenheim, and T. A. Niehaus, Phys. Rev. B 83, 245448 (2011).

${ }^{23}$ D. Dundas, E. J. McEniry, and T. N. Todorov, Nat. Nanotechnol. 4, 99 (2009).

${ }^{24}$ J.-T. Lü, M. Brandbyge, and P. Hedegård, Nano Lett. 10, 1657 (2010).

${ }^{25}$ J. Enkovaara, C. Rostgaard, J. J. Mortensen, J. Chen, M. Dułak, L. Ferrighi, J. Gavnholt, C. Glinsvad, V. Haikola, H. A. Hansen et al., J. Phys.: Condens. Matter 22, 253202 (2010).

${ }^{26}$ A. H. Larsen, M. Vanin, J. J. Mortensen, K. S. Thygesen, and K. W. Jacobsen, Phys. Rev. B 80, 195112 (2009).
${ }^{27}$ E. Louis, J. A. Vergés, J. J. Palacios, A. J. Pérez-Jiménez, and E. SanFabián, Phys. Rev. B 67, 155321 (2003).

${ }^{28}$ J. Taylor, H. Guo, and J. Wang, Phys. Rev. B 63, 245407 (2001).

${ }^{29}$ M. Brandbyge, J.-L. Mozos, P. Ordejón, J. Taylor, and K. Stokbro, Phys. Rev. B 65, 165401 (2002).

${ }^{30}$ Y. Xue, S. Datta, and M. A. Ratner, Chem. Phys. 281, 151 (2002).

${ }^{31}$ F. Guinea, C. Tejedor, F. Flores, and E. Louis, Phys. Rev. B 28, 4397 (1983).

${ }^{32}$ See, for example, H. Haug, and A.-P. Jauho, Quantum Kinetics in Transport and Optics of Semiconductors (Springer, New York, 1998).

${ }^{33}$ A. Kiejna, G. Kresse, J. Rogal, A. De Sarkar, K. Reuter, and M. Scheffler, Phys. Rev. B 73, 035404 (2006).

${ }^{34}$ Y. Meir and N. S. Wingreen, Phys. Rev. Lett. 68, 2512 (1992).

${ }^{35}$ K. S. Thygesen, Phys. Rev. B 73, 035309 (2006).

${ }^{36}$ J. Taylor, H. Guo, and J. Wang, Phys. Rev. B 63, 245407 (2001).

${ }^{37} \mathrm{R}$. Li, Ph.D. thesis, School of Electronics and Computer Science, Peking University, 2008.

${ }^{38}$ T. N. L. Patterson, Math. Comput. 22, 847 (1968).

${ }^{39}$ Q. Zekan, H. Shimin, L. Rui, S. Ziyong, Z. Xingyu, and X. Zengquan, J. Comput. Theor. Nanosci. 5, 671 (2008).

${ }^{40}$ T. Ozaki, K. Nishio, and H. Kino, Phys. Rev. B 81, 035116 (2010).

${ }^{41}$ M. Di Ventra, Y.-C. Chen, and T. N. Todorov, Phys. Rev. Lett. 92, 176803 (2004).

${ }^{42}$ J. B. Oostinga, H. B. Heersche, X. Liu, A. F.Morpurgo, and L. M. K. Vandersypen, Nat. Mater.7, 151 (2008).

${ }^{43}$ M. F.Craciun, S. Russo, M. Yamamoto, J. B. Oostinga, A. F. Morpurgo, and S. Tarucha, Nat. Nanotechnol. 4, 383 (2009).

${ }^{44}$ S. Kubatkin, A. Danilov, M. Hjort, J. Cornil, J.-L. Bredas, N. StuhrHansen, P. Hedegard, and T. Bjornholm, Nature (London) 425, 698 (2003).

${ }^{45}$ S. Hyunwook, K. Youngsang, J. Y. Hee, J. Heejun, M. A. Reed, and L. Takhee, Nature (London) 462, 1039 (2009).

${ }^{46}$ S. S. Datta, D. R. Strachan, and A. T. C. Johnson, Phys. Rev. B 79, 205404 (2009).

${ }^{47}$ M. Strange, O. Lopez-Acevedo, and H. Häkkinen, J. Phys. Chem. Lett. 1, 1528 (2010).

${ }^{48}$ P. D. Jadzinsky, G. Calero, C. J. Ackerson, D. A. Bushnell, and R. D. Kornberg, Science 318, 430 (2007).

${ }^{49}$ Y. Wang, Q. Chi, N. S. Hush, J. R. Reimers, J. Zhang, and J. Ulstrup, J. Phys. Chem. C 113, 19601 (2009).

${ }^{50}$ T. Markussen, J. Schiotz, and K. S. Thygesen, J. Chem. Phys. 132, 224104 (2010)

${ }^{51}$ T. Markussen, R. Stadler, and K. S. Thygesen, Nano Lett. 10, 4260 (2010).

${ }^{52}$ D. A. Areshkin and B. K. Nikolić, Phys. Rev. B 79, 205430 (2009).

${ }^{53}$ M. Fujita, K. Wakabayashi, K. Nakada, and K. Kusakabe, J. Phys. Soc. Jpn. 65, 1920 (1996).

${ }^{54}$ J. Zhang, S. Hou, R. Li, Z. Qian, R. Han, Z. Shen, X. Zhao, and Z. Xue, Nanotechnology 16, 3057 (2005). 